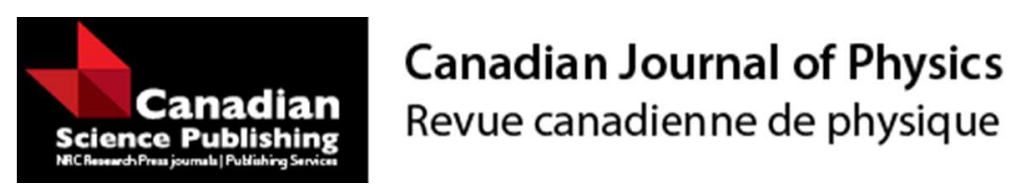

\title{
Attosecond source generation by using the polarized gating two-color field combined with the unipolar pulse
}

\begin{tabular}{|r|l|}
\hline Journal: & Canadian Journal of Physics \\
\hline Manuscript ID & cjp-2015-0711.R1 \\
\hline Danuscript Type: & Article \\
\hline Complete List of Authors: & $\begin{array}{l}\text { Feng, Liqiang; College of Science, Liaoning University of Technology } \\
\text { Liu, Hang; School of Chemical and Environmental Engineering, Liaoning } \\
\text { University of Technology }\end{array}$ \\
\hline Keyword: & $\begin{array}{l}\text { high order harmonic generation, attosecond pulse, polarized gating } \\
\text { scheme, unipolar pulse, two-color field }\end{array}$ \\
\hline &
\end{tabular}

\section{SCHOLARONE ${ }^{\text {m }}$}

Manuscripts 


\title{
Attosecond source generation by using the polarized gating two-color field combined with the unipolar pulse
}

Liqiang Feng and Hang Liu

\begin{abstract}
High-order harmonic generation and attosecond extreme-ultraviolet source generation have been theoretically investigated by controlling the two-color polarized gating field combined with the unipolar pulse. The results show that by properly optimizing the polarized two-color field as well as the unipolar pulse, not only the harmonic cutoff is remarkably extended, but also the single short quantum path has been selected to contribute to the harmonic spectrum, resulting in a $313 \mathrm{eV}$ supercontinuum with less modulated. Classical and quantum analyses are shown to explain the harmonic emission process. Finally, the proper superposition of harmonics, a series of isolated sub-50as pulses can be obtained.
\end{abstract}

Keywords: High-order harmonic generation; attosecond X-ray sources; Two-color polarized gating field; unipolar pulse

PACS Nos.: 42.65.Ky, 42.65.Re, 32.80.Fb

Liqiang Feng. College of Science, Liaoning University of Technology, Jinzhou, 121000, China;

State Key Laboratory of Molecular Reaction Dynamics, Dalian Institute of Chemical Physics, Chinese Academy of Sciences, Dalian, 116023, China

Hang Liu. School of Chemical and Environmental Engineering, Liaoning University of Technology, Jinzhou, 121000, China.

Corresponding author: Liqiang Feng (e-mail: lqfeng_lngy@126.com). 


\section{INTRODUCTION}

Attosecond extreme-ultraviolet pulse $\left(10^{-18} \mathrm{~s}\right)$ generation has been widely investigated due to its important applications in exploring and controlling the ultrafast dynamic processes in atoms and molecules [1-3]. High-order harmonic generation (HHG) as the most effective and the only method to experimentally generate isolated attosecond pulse has been intensively investigated during the last two decades [4-9].

Currently, the generation of the attosecond pulse in HHG can be well explained by the semi-classical 'three-step' model by Corkum [10]: where harmonics are emitted through the steps of tunneling ionization, acceleration and recombination. Usually, this process occurs at every half optical cycle of the laser field and there correspond two major electron paths for a given frequency of the emitted photon: the long quantum path (with earlier ionization and later recollision) and the short quantum path (with later ionization and earlier recollision) [11]. Each of them produces an attosecond pulse at every half-cycle, thus, an attosecond pulse train with four main bursts in an optical cycle is formed. However, due to the limitation of the harmonic cutoff energy $\mathrm{E}_{\max }=\mathrm{I}_{\mathrm{p}}+3.17 \mathrm{U}_{\mathrm{p}}$, where $\mathrm{I}_{\mathrm{p}}$ is the ionization potential and $U_{p}=I / 4 \omega^{2}$ is the ponderomotive energy of the free electron, the generated pulses are much longer than the natural time scale of the electronic process inside atoms and molecules. Moreover, for practical application, an isolated attosecond pulse is more useful as the attosecond-resolution probing and exploring tool. Therefore, how to enhance and select the harmonic emission becomes an interesting and important issue. So far, there are several successful methods to achieve this goal, for instance, the few-cycle pulse 
[12], the two-color or multi-color mixing method [13-15], the terahertz or the static field controlling scheme $[16,17]$, and the chirped pulse scheme $[18,19]$ etc.

Recently, an alternative technique, named unipolar pulse assisted generation of the isolated attosecond sources has attracted much attention [20-23]. A unipolar pulse is a field which consists of a short half-cycle followed by a long low-amplitude half cycle pulse of opposite polarity [20]. For instance, Orlando et al's theoretically obtain a 170 as pulse by using a weak $820 \mathrm{~nm}$ pulse and a controlling unipolar pulse [20]. Pan et al's theoretically obtain an isolated 38 as pulse by using the chirped pulse combined with the unipolar pulse [21] etc. However, the fundamental fields used in this scheme are almost the linear polarization. Little research has been conducted on the simultaneous combination of the polarization gating and the unipolar pulse. As we know, the harmonic emission is highly sensitive to the ellipticity of the laser pulse, thus, the time-varying polarization gating also plays a very important role in generating the isolated attosecond pulses [24-28]. For instance, Sansone et al. [24] obtained an isolated 130 as pulse by rapidly sweeping the laser polarization state within an optical cycle. Zhang et al. [25]. Zhao et al. [27] obtained a 67 as pulse by using the double optical gating method.

Thus, in this paper, to better understand the unipolar pulse effect on the generations of the harmonic spectra and the attosecond pulses, we further investigate harmonic extension and selection as well as the attosecond pulse generation by using the polarized gating two-color field combined with the unipolar pulse. It shows that by properly choosing the polarized gating two pulses and with the introduction of the 
unipolar pulse, an ultrabroad $313 \mathrm{eV}$ bandwidth with the dominant short quantum path contribution has been obtained, which can support a series of isolated sub-50as pulses.

\section{THEORY}

We perform time-dependent Schrödinger equation (TDSE) simulations in the single-active electron approximation in a two-dimensional (2D) model for the $\mathrm{He}$ atom with various polarizations. In the dipole approximation and the length gauge, the TDSE is given by (atomic units (a.u.) are used throughout this paper unless stated otherwise),

$$
i \frac{\partial \psi(x, y, t)}{\partial t}=\left[-\frac{1}{2} \frac{\partial^{2}}{\partial x^{2}}-\frac{1}{2} \frac{\partial^{2}}{\partial y^{2}}+V(x, y)+x E_{x}(t)+y E_{y}(t)\right] \psi(x, y, t),
$$

where $V(x, y)=-1.0 / \sqrt{x^{2}+y^{2}+0.07}$ is the soft Coulomb potential to match the ionization potential of $24.6 \mathrm{eV}$ for the ground state of He. $\mathrm{x}$ and $\mathrm{y}$ are the two-dimensional (2D) electronic coordinates, which are defined by -250 a.u. $<\mathrm{x}, \mathrm{y}<$ 250 a.u. with $\Delta x=\Delta y=0.5$ a.u.. The absorbing regions on $\mathrm{x}$ and $\mathrm{y}$ extend over the last 100 grid points. The time space is chosen to be $\Delta t=0.1$ a.u.. Propagation of the time-dependent electronic wave function $\psi(x, y, t)$ can be carried out using the standard second-order split-operator method [29-33]. The synthesized laser field can be expressed as $\vec{E}(t)=\vec{E}_{T}(t)+E_{u n i}(t)$. In particular, $\vec{E}_{T}(t)$ means the polarized two-color field which can be expressed as,

$$
\begin{aligned}
\vec{E}_{T}(t) & =\left[E_{1} \exp \left[-4 \ln (2) t^{2} / \tau_{1}^{2}\right] \cos \left(\omega_{1} t\right)+E_{2} \exp \left[-4 \ln (2) t^{2} / \tau_{2}^{2}\right] \cos (\theta) \cos \left(\omega_{2} t+\varphi\right)\right] \widehat{x} \\
& +E_{2} \exp \left[-4 \ln (2) t^{2} / \tau_{2}^{2}\right] \sin (\theta) \cos \left(\omega_{2} t+\varphi\right) \hat{y}
\end{aligned}
$$

Here $E_{i}, \omega_{i}$ and $\tau_{i}(i=1,2)$ are the amplitudes, the frequencies and the pulse durations 
of the $5 \mathrm{fs} / 800 \mathrm{~nm}$ and $10 \mathrm{fs} / 1600 \mathrm{~nm}$ pulses. $\theta$ and $\varphi$ are the polarized angle and the relative phase between the two pulses. While, the unipolar pulse is defined as,

$$
E_{u n i}(t)=k \xi\left(t-t_{0}^{\prime}\right) E_{i}\left[\frac{a\left(t-t_{0}^{\prime}\right)^{3} \exp \left[-8\left(t-t_{0}^{\prime}\right) / \tau_{u n i}\right]}{\tau_{u n i}^{3}}-\frac{b\left(t-t_{0}^{\prime}\right)^{5} \exp \left[-\left(t-t_{0}^{\prime}\right) / \tau_{u n i}\right]}{\tau_{u n i}^{5}}\right]
$$

where $\xi(t)$ is the step function, the unipolar pulse starts at $t_{0}$ and $\tau_{\text {uni }}$ is the pulse duration of the unipolar pulse. The parameters $a, b$ are chosen to be $a=400$ and $\mathrm{b}=10^{-5} \mathrm{a}$, respectively. $\mathrm{k}$ is the relative strength ratio of the unipolar pulse, and $\mathrm{kE}_{\mathrm{i}}$ $(i=1,2)$ is the pulse intensity of the unipolar pulse. Recent investigation shows that the unipolar pulse can be obtained from the resonant propagation of a few-cycle pulse through asymmetrical media with periodic sub-wavelength structure [34].

According to the Ehrenfest theorem [35], the time-dependent dipole acceleration can be written in the form,

$$
\begin{aligned}
& a(t)=-\langle\psi(x, y, t)|\nabla \vec{V}(x, y)+\vec{E}(t)| \psi(x, y, t)\rangle \\
& =-\left\langle\psi(x, y, t)\left|\left(\frac{\partial V(x, y)}{\partial x}+E_{x}(t)\right) \vec{e}_{x}+\left(\frac{\partial V(x, y)}{\partial y}+E_{y}(t)\right) \vec{e}_{y}\right| \psi(x, y, t)\right\rangle . \\
& =a_{x}(t) \vec{e}_{x}+a_{y}(t) \vec{e}_{y}
\end{aligned}
$$

The HHG spectra can be obtained by Fourier transforming the time-dependent dipole acceleration $\mathrm{a}(\mathrm{t})$,

$$
\begin{aligned}
S\left(\omega_{1}\right) & \sim\left|\int \exp \left(-i \omega_{1} t\right) a(t) d t\right|^{2} \\
& =\left|\int \exp \left(-i \omega_{1} t\right)\left(a_{x}(t) \vec{e}_{x}+a_{y}(t) \vec{e}_{y}\right) d t\right|^{2} \\
& \sim S_{x}\left(\omega_{1}\right)+S_{y}\left(\omega_{1}\right)
\end{aligned}
$$

Finally, the attosecond pulse can be obtained by harmonic superposing as follows, 


$$
I(t)=\left|\sum_{q} a_{q} e^{i q \omega_{1} t}\right|^{2},
$$

where $a_{q}=\int a(t) e^{-i q \omega_{1} t} d t$

\section{RESULTS AND DISCUSSION}

Fig. 1(a) shows the HHG spectra driven by the polarized two-color field with $\theta=0.0 \pi$ (solid black line), $\theta=0.2 \pi$ (solid red line), $\theta=0.4 \pi$ (solid blue line and this is what we find the optimal value for harmonic extension under the present laser field) and $\theta=0.6 \pi$ (solid dark cyan line), respectively. The other parameters are chosen to be $5 \mathrm{fs} / 800 \mathrm{~nm}, \quad \mathrm{I}_{1}=1.0 \times 10^{15} \mathrm{~W} / \mathrm{cm}^{2}$ and $10 \mathrm{fs} / 1600 \mathrm{~nm}, \quad \mathrm{I}_{2}=1.0 \times 10^{14} \mathrm{~W} / \mathrm{cm}^{2}, \quad \varphi=5 / 6 \pi$, respectively. It shows that the harmonic spectra are very sensitive to the polarized angle of the two-color pulse. In particular, (i) with the introduction of the polarized angle $\theta$, the harmonic cutoff is enhanced resulting in two harmonic plateaus, however, the intensity of the second harmonic plateau is too weak in comparison with the first one, which is unbeneficial to produce the high intense attosecond pulse. Thus, we only consider the first harmonic cutoff for attosecond pulse generation. (ii) With the increasing of the polarized angle $\theta$, the harmonic cutoff is inversely decreased, but the modulation on the harmonic plateau is also reduced, which is beneficial to the harmonic selection and the isolated attosecond pulse generation. Thus, through our calculations, the HHG spectrum from $\theta=0.4 \pi$ is the optimal polarized angle in the present synthesized field. Fig. 1(b) shows the harmonic spectrum in $\mathrm{x}\left(\mathrm{E}_{\mathrm{x}}\right)$ and $\mathrm{y}\left(\mathrm{E}_{\mathrm{y}}\right)$ components for the case of the two-color polarized pulse with $\theta=0.4 \pi$. As we see, the HHG have contributions in both $\mathrm{x}$ and $\mathrm{y}$ components, thus, the ellipticity will be 
generated in the harmonic emission process. According to Zhou et al.'s [36] investigation, we know that the ellipticity of the harmonic emission can be defined as $\xi=\sqrt{E_{\min o r} / E_{\text {major }}}$, where $\mathrm{E}_{\text {minor }}$ and $\mathrm{E}_{\text {major }}$ are the intensity of the minor and major axis of the HHG in $\mathrm{x}$ and $\mathrm{y}$ directions and the corresponding results are shown in Fig. 1(c). Clearly, due to the strong harmonic oscillation in the low harmonic orders, the harmonic emission spectrum can be strongly elliptically polarized. However, for the high harmonic orders, especially for the plateau region, due to the steady harmonic intensities in $\mathrm{x}$ and $\mathrm{y}$ directions, the especially of the harmonic almost equals to a fixed value (i.e. $\varepsilon \approx 0.3$ )

To explain the harmonic extension process, in Fig. 2, we present the laser profiles of the above four laser fields. Firstly, we see the laser profile of the two-color field with $\theta=0.0 \pi$ as shown in Fig. 2(a) (corresponding to the linear two-color field). For comparison, the single two color pulses with $800 \mathrm{~nm}$ and $1600 \mathrm{~nm}$ have also been shown in Fig. 2(a). According to the 'three-step' model [10], we know that the electron can be ionized around the A point and accelerated away from the ion core. Further, when the electric field inverses its direction around the B point, the electron is first decelerated and then accelerated reversely. Finally, the electron can recombine with its parent ion at the $\mathrm{C}$ point and emit a harmonic photon. From analyzing the laser profiles, we see that due to the introduced second controlling $1600 \mathrm{~nm}$ pulse, the B-C process has been reduced. Thus, the electron must take much less time in its processes of accelerating and returning to the parent ion, which is unbeneficial to harmonic extension. Thus, the present linear two-color field $(\theta=0.0 \pi)$ is not suitable 
for harmonic extension and attosecond pulse generation. Fig. 2(b1) shows the laser profiles of $\mathrm{x}$ and $\mathrm{y}$ components for the case of the two-color field with $\theta=0.2 \pi$. Clearly, we see that due to the polarized gating effect, the amplitude B-C process in the $\mathrm{x}$ direction has been enhanced in comparison with the single $800 \mathrm{~nm}$ pulse, the single $1600 \mathrm{~nm}$ pulse and the two-color field with $\theta=0.0 \pi$. According to the 'three-step' model, we know that the maximum harmonic value $\left(\mathrm{E}_{\max }=\mathrm{I}_{\mathrm{p}}+\mathrm{nU} \mathrm{U}_{\mathrm{p}}, \mathrm{U}_{\mathrm{p}}=\mathrm{I}^{2} / 4 \omega^{2}\right)$ is decided by either the amplitude intensities (relating to the pulse intensities I) or the widths (relating to the frequencies $\omega$ ). Due to the enhanced amplitude intensity, the ponderomotive energy $U_{p}$ has been enhanced, which is the main reason behind the harmonic extension shown in Fig. 1(a). However, with the increasing of the polarized angle, we see that the amplitudes $\mathrm{B}-\mathrm{C}$ processes in the $\mathrm{x}$ direction have been reduced as shown in Figs. 2(c1) and (d1) for the cases of the two-color field with $\theta=0.4 \pi$ and $\theta=0.6 \pi$, which is responsible for the decreasing harmonic cutoff shown in Fig. 1(a). Figs. 2(b2)-(d2) show the 3D laser profiles of the above two-color field with $\theta=0.2 \pi$, $\theta=0.4 \pi$ and $\theta=0.6 \pi$, respectively. Clearly, they show on a quick glance that the rotating fields have a very different appearance, which is the probable reason for the reducing modulation on the harmonic spectra [37].

Fig. 3(a) shows the HHG spectra driven by the above optimal two-color polarized field $(\theta=0.4 \pi)$ and an introduced unipolar pulse in $\mathrm{x}$ direction. Here, the unipolar pulse is chosen to be $\mathrm{k}=0.1, \tau_{\text {uni }}=5.0 \mathrm{fs}$ with different starting position $\mathrm{t}_{0}$. Clearly, with the introduction of the unipolar pulse in $\mathrm{x}$ direction, the harmonic cutoff has been further extended. Especially for $t_{0}=4.4 T_{1}$ case $\left(T_{1}\right.$ means the optical cycle of 
the $800 \mathrm{~nm}$ pulse), an ultrabroad $313 \mathrm{eV}$ bandwidth with less modulation has been obtained. Fig. 3(b) shows the harmonic spectra from the two-color polarized field combined with the unipolar pulse in y direction. The results show that by properly adding a unipolar pulse in y direction, the harmonic cutoff can also be enhanced but with a smaller degree in comparison with adding unipolar pulse in $\mathrm{x}$ direction. Thus, we see that by adding the unipolar pulse to the two-color polarized field in $\mathrm{x}$ direction with $\mathrm{t}_{0}=4.4 \mathrm{~T}_{1}$ is much better for harmonic emission. Figs. $3(\mathrm{c})$ and $(\mathrm{d})$ show the HHG spectra in $\mathrm{x}$ and $\mathrm{y}$ components for the cases of adding unipolar pulse in $\mathrm{x}$ and $\mathrm{y}$ directions with $\mathrm{t}_{0}=4.4 \mathrm{~T}_{1}$ and $\mathrm{t}_{0}=5.2 \mathrm{~T}_{1}$, respectively. It shows that whether introduced the unipolar pulse in $\mathrm{x}$ or $\mathrm{y}$ direction, the harmonics in $\mathrm{x}$ direction always play the dominating role in harmonic spectra, and the harmonic intensities, especially for the harmonic plateau region, are similar as those generated from the no unipolar pulse case shown in Fig. 1(b).

To understand the effect of the unipolar pulse on the harmonic emission, in Fig. 4(a), we present the laser profile of the single unipolar pulse with $\mathrm{k}=0.1, \tau_{\text {uni }}=5.0 \mathrm{fs}$ and $t_{0}=4.4 \mathrm{~T}_{1}$. It shows that the unipolar pulse is a half cycle pulse. Thus, it has some advantages for varying the laser profile of the combined field. For instance, with the introduction of the uinpolar pulse, the laser profile of the combined field can be changed in the appointed position and the others can not be changed. This is a very beneficial information. As we know that the harmonic cutoff is relevant to the amplitude of the pulse. Thus, if we properly adding a unipolar to enhanced the amplitude of the pulse, then, the harmonic cutoff can be extended. And this is the 
main reason behind the harmonic extension with the introduction of the unipolar pulse shown in Fig. 3. To confirm the above illustration, the laser profiles of the $x$ and $y$ components for the combined fields by adding the unipolar pulse in $\mathrm{x}$ and $\mathrm{y}$ directions with $\mathrm{t}_{0}=4.4 \mathrm{~T}_{1}$ and $\mathrm{t}_{0}=5.2 \mathrm{~T}_{1}$ have been shown in Figs. $4(\mathrm{~b} 1)$ and (c1), respectively. Clearly, with the proper introduction of the unipolar pulses in $\mathrm{x}$ or $\mathrm{y}$ directions, the amplitudes (B-C process) of the corresponding pulse $E_{x}(t)$ or $E_{y}(t)$ have been enhanced, thus leading to the extension of the ponderomotive energy and is responsible for the harmonic extension. Figs. 4(b2) and (c2) show the corresponding 3D laser profiles of the above two combined field. It is found the although the rotation of the combined fields produce almost no change, the corresponding amplitudes have been enhanced with the introduction of the corresponding unipolar pulses.

To better understand the harmonic emission process, in Fig. 5, we present the time-frequency distributions of the harmonic spectra, obtained by using the wavelet transformation of the dipole acceleration a(t) [38,39],

$$
A\left(t, \omega_{1}\right)=\int a\left(t^{\prime}\right) \sqrt{\omega_{1}} W\left(\omega_{1}\left(t^{\prime}-t\right)\right) d t^{\prime},
$$

where $W\left(\omega_{1}\left(t^{\prime}-t\right)\right)$ is the Morlet wavelet with the formula of,

$$
W(\xi)=\left(\frac{1}{\sqrt{\alpha}}\right) e^{i \xi} e^{-\xi^{2} / 2 \alpha^{2}}
$$

here $\alpha=30$ in our calculations. For the linearly-polarized two-color field case $(\theta=0.0 \pi)$, as shown in Fig. 5(a), we see that there are many emission bursts on the harmonic emission process, and the maximum harmonic cutoff is agreement well with the quantum result shown in Fig. 1(a). Moreover, each burst receives similar contributions from the long quantum path (right path) having earlier ionization and 
later recollision and the short quantum path (left path) with later ionization but earlier recollision, which is responsible for the large interference on the harmonics [11]. While with the introduction of the polarized angle, as shown in Fig. 5(b) for the case of $\theta=0.4 \pi$, the maximum emission burst is enhanced compared with the linearly-polarized two-color case. This is because that due to the enhancement of the amplitude and the varieties of the laser field as illustrated before, the electron must take much more time in its process of accelerating and returning to the parent ion, thus, resulting in the enhancing of the maximum harmonic emission burst. Fig. 5(c) shows the time-frequency harmonic spectrum of the two-color polarized pulse combined with the optimal unipolar pulse (two-color polarized pulse+unipolar pulse in $\mathrm{x}$ direction with $\mathrm{t}_{0}=4.4 \mathrm{~T}_{1}$ ), we see that with the introduction of the unipolar pulse, not only the maximum harmonic burst is remarkably enhanced, but also the single short quantum path is selected for the harmonic emission process, which is responsible for the small interference on the plateau and is beneficial to generate an isolated attosecond source.

Fig. 6(a) shows the relative strength ratio (k) effect on the harmonic emission. The other parameters are the same as those in Fig. 1(a) solid blue line. As illustrated before, the maximum harmonic cutoffs $\left(\mathrm{E}_{\mathrm{max}}=\mathrm{I}_{\mathrm{p}}+\mathrm{nU}_{\mathrm{p}}\right)$ are relevant to the amplitude intensities of the laser profile. Thus, with the increasing of the relative strength ratio, the harmonic cutoff has been further extended, but the large interference structures on the harmonic are unbeneficial to isolated attosecond pulse generation. Fig. 6(b) shows the controlling pulse duration $\tau_{\text {uni }}$ effect on the harmonic emission. Clearly, by 
properly choosing the starting positions $\mathrm{t}_{0}$ of the unipolar pulse, the similar harmonic extension and selection can also be achieved from different pulse duration $\tau_{\text {uni. }}$. Moreover, with the increasing of the pulse duration $\tau_{\text {uni, }}$, the starting positions $\mathrm{t}_{0}$ of the unipolar pulse is forward. Thus, through the above calculations, we choose $\mathrm{k}=0.1$ and $\tau_{\text {uni }}=5.0 \mathrm{fs}$ as a proper controlling unipolar pulse and a supercontinuum with the $313 \mathrm{eV}$ bandwidth can be obtained, which will favorite to support the isolated attosecond pulse.

Fig. 7 shows the temporal profiles of the attosecond pulses. In particular, by respectively superposing the harmonics of the above optimal synthesized field case (Fig. 1(a) solid blue line) from the 50th to the 90th orders (photon energies between $78 \mathrm{eV}$ and $140 \mathrm{eV}$ ), from the 90 th to the 130 th orders (photon energies between $140 \mathrm{eV}$ and $201 \mathrm{eV}$ ), from the 130th to the 170th orders (photon energies between $201 \mathrm{eV}$ and $264 \mathrm{eV}$ ), and from the 170th to the 210th orders (photon energies between $264 \mathrm{eV}$ and $326 \mathrm{eV}$ ), four isolated extreme-ultraviolet pulses with durations of 47as, 40as, 33as and 36as can be obtained, as shown in Figs. 7(a)-(d), respectively.

\section{CONCLUSIONS}

In conclusion, we have theoretically investigated the generations of the harmonics and the attosecond extreme-ultraviolet pulses in the presence of the two-color polarized gating pulse combined with a unipolar pulse. The results show that by properly choosing the polarized gating two-color field and the unipolar pulse (polarized two-color field with $\theta=0.4 \pi, \varphi=5 / 6 \pi$ combined with a unipolar pulse in $\mathrm{X}$ 
direction with $\mathrm{k}=0.1$ and $\tau_{\mathrm{uni}}=5.0 \mathrm{fs}$ ), not only the harmonic cutoff has been remarkably enhanced, resulting in a $313 \mathrm{eV}$ bandwidth, but also the single short quantum path has been selected to contribute to the harmonic emission. As a result, a series of isolated attosecond pulses with durations of sub-50as can be obtained.

\section{ACKNOWLEDGMENTS}

The authors thank Professor Keli Han for providing us the computational code used in the present work. This work was supported by National Science Foundation of China (No. 11504151), the doctoral scientific research foundation of Liaoning province (No. 201501123), and the scientific research fund of Liaoning provincial education department (No. L2014242).

\section{REFERENCES}

[1] T. Brabec and F. Krausz, Rev. Mod. Phys. 72, 545 (2000).

[2] F. Krausz and M. Ivanov, Rev. Mod. Phys. 81, 163 (2009).

[3] L. Q. Feng and H. Liu, Can. J. Phys. 92, 1592 (2014).

[4] G. T. Zhang, J. Wu, C. L. Xia and X. S. Liu, Phys. Rev. A 80, 055404 (2009).

[5] B. Kim, J. Ahn, Y. L. Yu, Y. Cheng, Z. Z. Xu and D. E. Kim, Opt. Express 16, 10331 (2008).

[6] Z. Zhai, R. F. Yu, X. S. Liu and Y. J. Yang, Phys. Rev. A 78, 041402 (2008).

[7] P. Zou, Z. N. Zeng, Y. H. Zheng, Y. Y. Lu, P. Liu, R. X. Li and Z. Z. Xu, Phys. Rev. 
A 81, 033428 (2010).

[8] Y. H. Wang, C. Yu, Q. Shi, Y. D. Zhang, X. Cao, S. C. Jiang and R. F. Lu, Phys. Rev. A 89, 023825 (2014).

[9] L. X. He, P. F. Lan, C. Y. Zhai, Y. Li, Z. Wang, Q. B. Zhang and P. X. Lu, Phys. Rev. A 91, 023428 (2015).

[10] P. B. Corkum, Phys. Rev. Lett. 71, 1994 (1993).

[11] Y. Mairesse, A. D. Bohan, L. J. Frasinski, H. Merdji, L. C. Dinu, P. Monchicourt, P. Breger, M. Kovačev, R. Taïeb, B. Carré, H. G. Muller, P. Agostini and P. Salières, Science 302, 1540 (2003).

[12] E. Goulielmakis, M. Schultze, M. Hofstetter, V. S. Yakovlev, J. Gagnon, M. Uiberacker, A. L. Aquila, E. M. Gullikson, D. T. Attwood, R. Kienberger, F. Krausz and U. Kleineberg, Science 320, 1614 (2008).

[13] Z. Zeng, Y. Cheng, X. Song, R. Li and Z. Xu, Phys. Rev. Lett. 98, 203901 (2007).

[14] P. F. Lan, P. X. Lu, W. Cao, Y. H. Li and X. L. Wang, Phys. Rev. A 76, 011402 (2007).

[15] L. Q. Feng, Y. B. Duan and T. S. Chu, Ann. Phys. (Berlin) 525, 915 (2013).

[16] G. J. Zhao, X. L. Guo, T. J. Shao and K. Xue, New J. Phys. 13, 093035 (2011).

[17] L. Q. Feng and T. S. Chu, Chem. Phys. 405, 26 (2012).

[18] L. Q. Feng and T. S. Chu, Phys. Rev. A 84, 053853 (2011).

[19] J. Wu, G. T. Zhang, C. L. Xia and X. S. Liu, Phys. Rev. A 82, 013411 (2012).

[20] G. Orlando, P. P. Corso, E. Fiordilino and F. Persico, J. Mod. Opt. 56, 1761 
(2009).

[21] Y. Pan, S. F. Zhao and X. X. Zhou, Phys. Rev. A 87, 035805 (2013).

[22] G. Orlando, P. P. Corso and E. Fiordilino, J. Mod. Opt. 57, 2069 (2010).

[23] X. H. Song, W. F. Yang, Z. N. Zeng, R. X. Li and Z. Z. Xu, Phys. Rev. A 82, 053821 (2010).

[24] G. Sansone et al., Science 314, 443 (2006).

[25] Z. H. Chang, Phys. Rev. A 71, 023813 (2005).

[26] E. Cunningham and Z. H. Chang, IEEE J. Sel. Top. Quant. 21, 8700806 (2015).

[27] K. Zhao, Q. Zhang, M. Chini, Y. Wu, X. W. Wang and Z. H. Chang, Opt. Lett. 37, 3891 (2012).

[28] L. Q. Feng, M. H. Yuan and T. S. Chu, Phys. Plasmas 20, 122307 (2013).

[29] J. Hu, K. L. Han and G. Z. He, Phys. Rev. Lett. 95, 123001 (2005).

[30] T. S. Chu, Y. Zhang and K. L. Han, Int. Rev. Phys. Chem. 25, 201 (2006).

[31] L. Q. Feng and H. Liu, Phys. Plasmas. 22, 013107 (2015).

[32] W. L. Li and K. L. Han, J. Math. Chem. 51, 1293 (2013).

[33] R. F. Lu, P. Y. Zhang and K. L. Han, Phys. Rev. E 77, 066701 (2008).

[34] X. H. Song, W. F. Yang, Z. N. Zeng, R. X. Li and Z. Z. Xu, Phys. Rev. A 82, 053821 (2010).

[35] K. Burnett, V. C. Reed, J. Cooper and P. L. Knight, Phys. Rev. A 45, 3347 (1992).

[36] X. B. Zhou, R. Lock, N. Wagner, W. Li, H. C. Kapteyn and M. M. Murnane, Phys. Rev. Lett. 102, 073902 (2009)

[37] L. Q. Feng, H. Liu and X. J. Liu, J. Math. Chem. 52, 2074 (2014). 
[38] P. Antoine, B. Piraux and A. Maquet, Phys. Rev. A 51, R1750 (1995).

[39] L. Q. Feng and T. S. Chu, J. Chem. Phys. 136, 054102 (2012). 


\section{Figure Captions}

Fig. 1 (Color online) (a) HHG spectra of the two-color polarized pulse with $\theta=0.0 \pi$ (solid black line), $\theta=0.2 \pi$ (solid red line), $\theta=0.4 \pi$ (solid blue line) and $\theta=0.6 \pi$ (solid dark cyan line). The laser parameters are chosen to be $5 \mathrm{fs} / 800 \mathrm{~nm}, \mathrm{I}_{1}=1.0 \times 10^{15} \mathrm{~W} / \mathrm{cm}^{2}$ and $10 \mathrm{fs} / 1600 \mathrm{~nm}, \mathrm{I}_{2}=1.0 \times 10^{14} \mathrm{~W} / \mathrm{cm}^{2}, \varphi=5 / 6 \pi$. (b) HHG spectra and the $\mathrm{x}$ and $\mathrm{y}$ components for the case of the two-color polarized pulse with $\theta=0.4 \pi$. (c) Ellipticity $\varepsilon$ of the harmonic emission for the case of the two-color polarized pulse with $\theta=0.4 \pi$.

Fig. 2 (Color online) (a) Laser profiles of the single $800 \mathrm{~nm}$, single $1600 \mathrm{~nm}$ and the two-color polarized pulse with $\theta=0.0 \pi$. (b1)-(d1) Laser profiles in the $\mathrm{x}$ and $\mathrm{y}$ components for the cases of the two-color polarized pulse with $\theta=0.2 \pi, \theta=0.4 \pi$ and $\theta=0.6 \pi$, respectively. (b2)-(d2) $3 \mathrm{D}$ laser profiles of the two-color polarized pulse with $\theta=0.2 \pi, \theta=0.4 \pi$ and $\theta=0.6 \pi$, respectively. $T_{1}$ means the optical cycle of $800 \mathrm{~nm}$ pulse in all the following figures unless stated otherwise.

Fig. 3 (Color online) (a) HHG spectra from the two-color polarized pulse $(\theta=0.4 \pi)$ combined with the unipolar pulse in $\mathrm{x}$ direction. The unipolar pulse is chosen to be $\mathrm{k}=0.1, \tau_{\text {uni }}=5.0 \mathrm{fs}$ with $\mathrm{t}_{0}=4.0 \mathrm{~T}_{1}($ solid black line $), 4.4 \mathrm{~T}_{1}$ (solid red line), $4.7 \mathrm{~T}_{1}$ (solid blue line) and $4.9 \mathrm{~T}_{1}$ (solid dark cyan line). (b) The similar results as those in Fig. 3(a), but the unipolar pulse is added in y direction with $\mathrm{t}_{0}=4.4 \mathrm{~T}_{1}$ (solid black line), $5.2 \mathrm{~T}_{1}$ (solid red line) and 5.5 $\mathrm{T}_{1}$ (solid blue line). (c) $\mathrm{HHG}$ spectra and the $\mathrm{x}$ and $\mathrm{y}$ components for the case of adding the unipolar pulse in $\mathrm{x}$ direction with $\mathrm{t}_{0}=4.4 \mathrm{~T}_{1}$. (d) HHG spectra and the $\mathrm{x}$ and $\mathrm{y}$ components for the case of adding the unipolar pulse in $\mathrm{y}$ direction with $\mathrm{t}_{0}=5.2 \mathrm{~T}_{1}$. 
Fig. 4 (Color online) (a) Laser profile of the single unipolar pulse with $k=0.1$, $\tau_{\text {uni }}=5.0 \mathrm{fs}$ and $\mathrm{t}_{0}=4.4 \mathrm{~T}_{1}$. (b1) and (c1) Laser profiles in the $\mathrm{x}$ and $\mathrm{y}$ components for the cases of adding the unipolar pulse in $\mathrm{x}$ and $\mathrm{y}$ directions with $\mathrm{t}_{0}=4.4 \mathrm{~T}_{1}$ and $\mathrm{t}_{0}=5.2 \mathrm{~T}_{1}$, respectively. (b2) and (c2) 3D laser profiles for the above two cases.

Fig. 5 (Color online) Time-frequency harmonic distributions for (a) the two-color polarized pulse with $\theta=0.0 \pi$; (b) the two-color polarized pulse with $\theta=0.4 \pi$; (c) the two-color polarized pulse $(\theta=0.4 \pi)$ combined with the unipolar pulse in $\mathrm{x}$ direction with $\mathrm{t}_{0}=4.4 \mathrm{~T}_{1}$.

Fig. 6 (Color online) (a) HHG spectra from the two-color polarized pulse $(\theta=0.4 \pi)$ combined with the unipolar pulse in $\mathrm{x}$ direction with $\tau_{\text {uni }}=5.0 \mathrm{fs}, \mathrm{t}_{0}=4.4 \mathrm{~T}_{1}, \mathrm{k}=0.1$ (solid black line) and $\mathrm{k}=0.3$ (solid red line). (b) HHG spectra from the two-color polarized pulse $(\theta=0.4 \pi)$ combined with the unipolar pulse in $\mathrm{x}$ direction with $\mathrm{k}=0.1, \tau_{\mathrm{uni}}=3.0 \mathrm{fs}$, $\mathrm{t}_{0}=4.7 \mathrm{~T}_{1}$ ( solid black line), $\mathrm{k}=0.1, \tau_{\text {uni }}=5.0 \mathrm{fs}, \mathrm{t}_{0}=4.4 \mathrm{~T}_{1}$ (solid red line) and $\mathrm{k}=0.1$, $\tau_{\text {uni }}=7.0 \mathrm{fs}, \mathrm{t}_{0}=4.2 \mathrm{~T}_{1}$ ( solid blue line).

Fig. 7 (Color online) The temporal profiles of the attosecond pulses by superposing harmonics of the optimal synthesized field case (a) from the 50th to the 90th orders, (b) from the 90th to the 130th orders, (c) from the 130th to the 170th orders and (d) from the 170 th to the 210 th orders. 

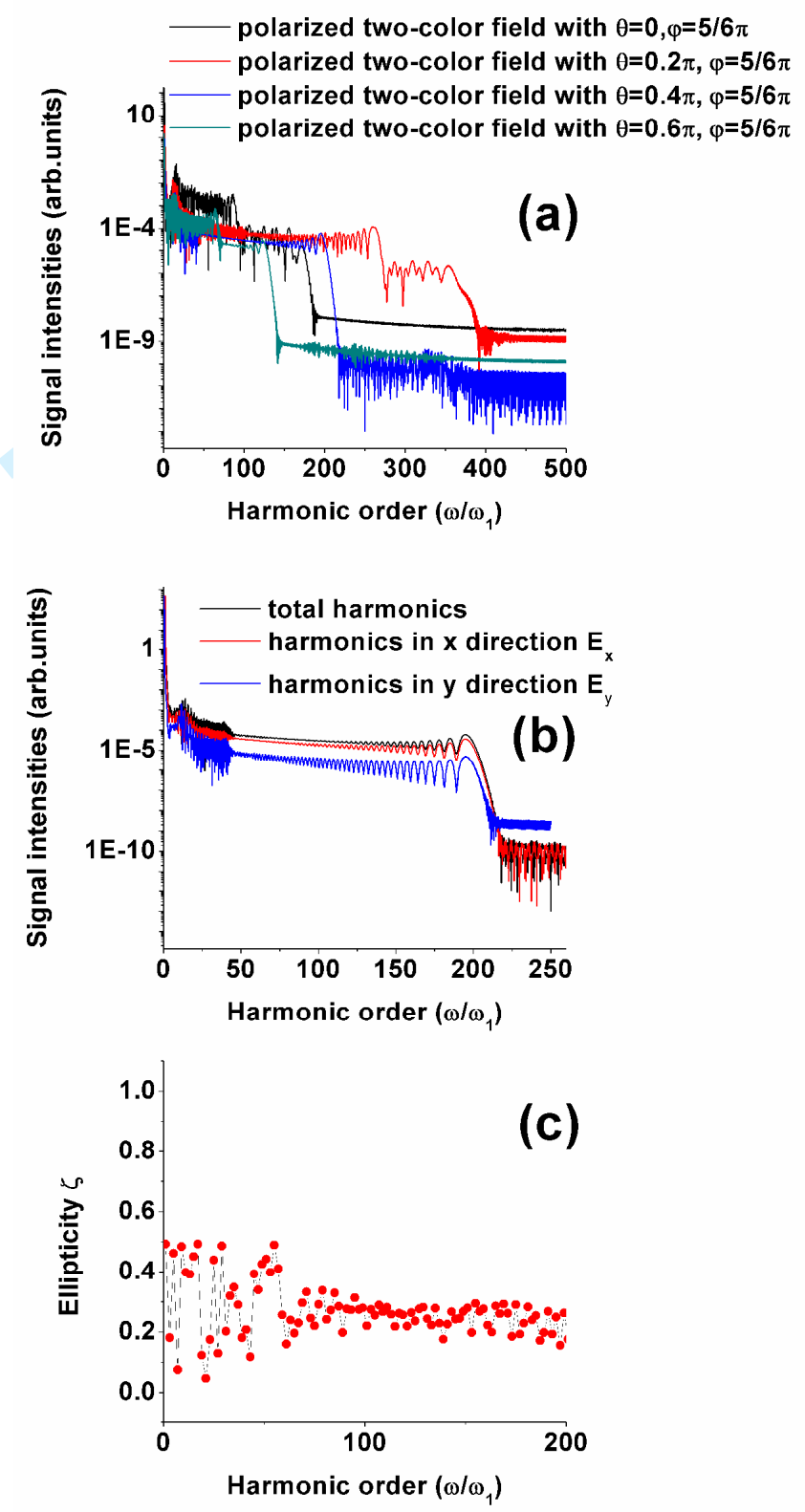

Fig. 1 

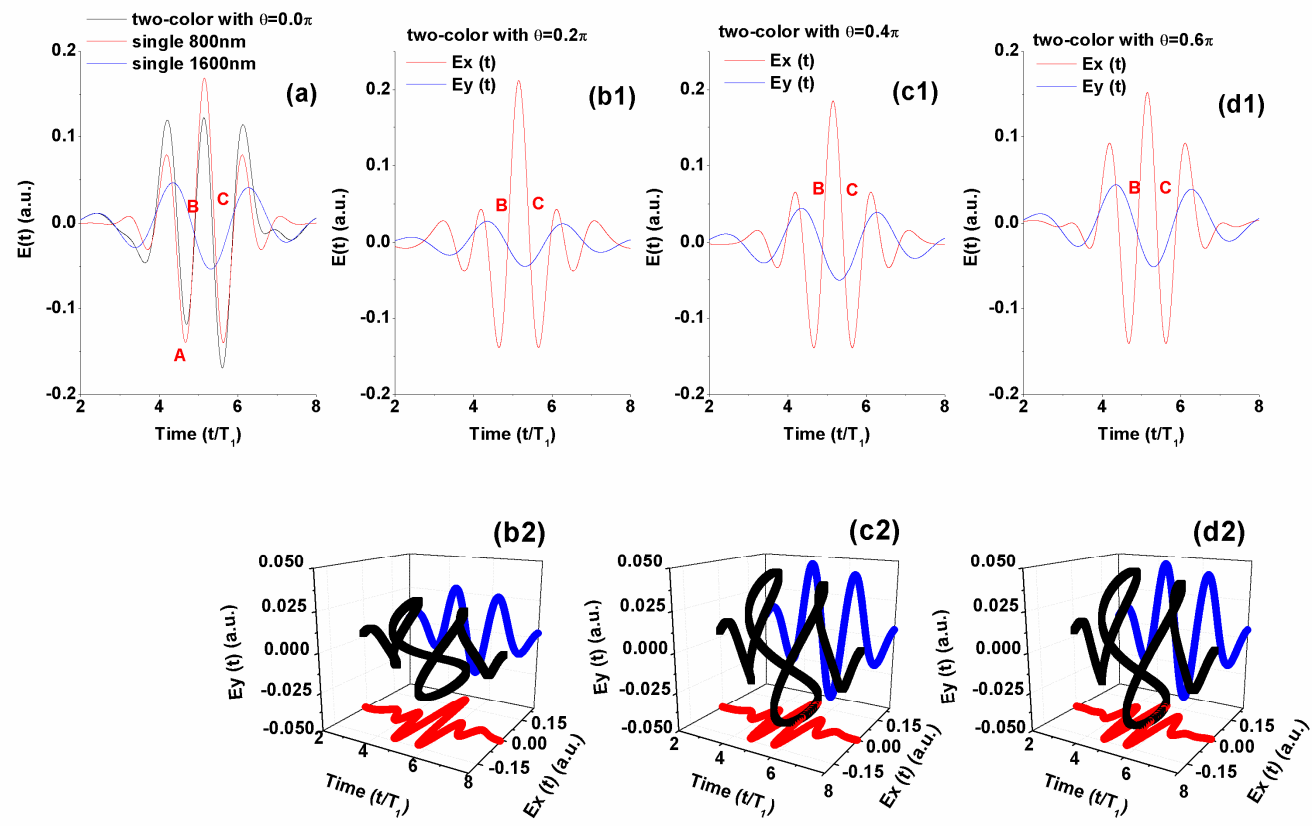

Fig. 2 

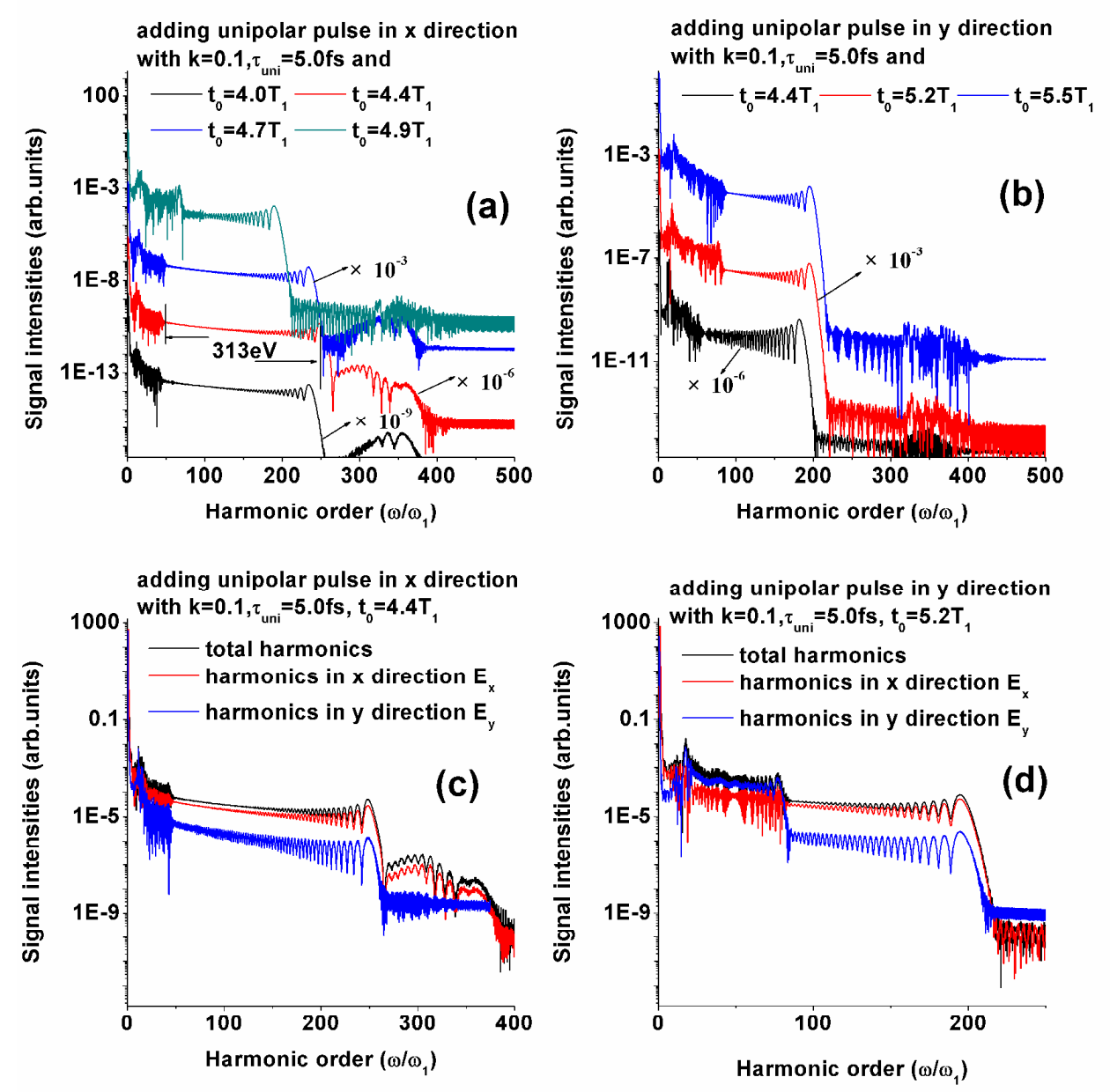

Fig. 3 

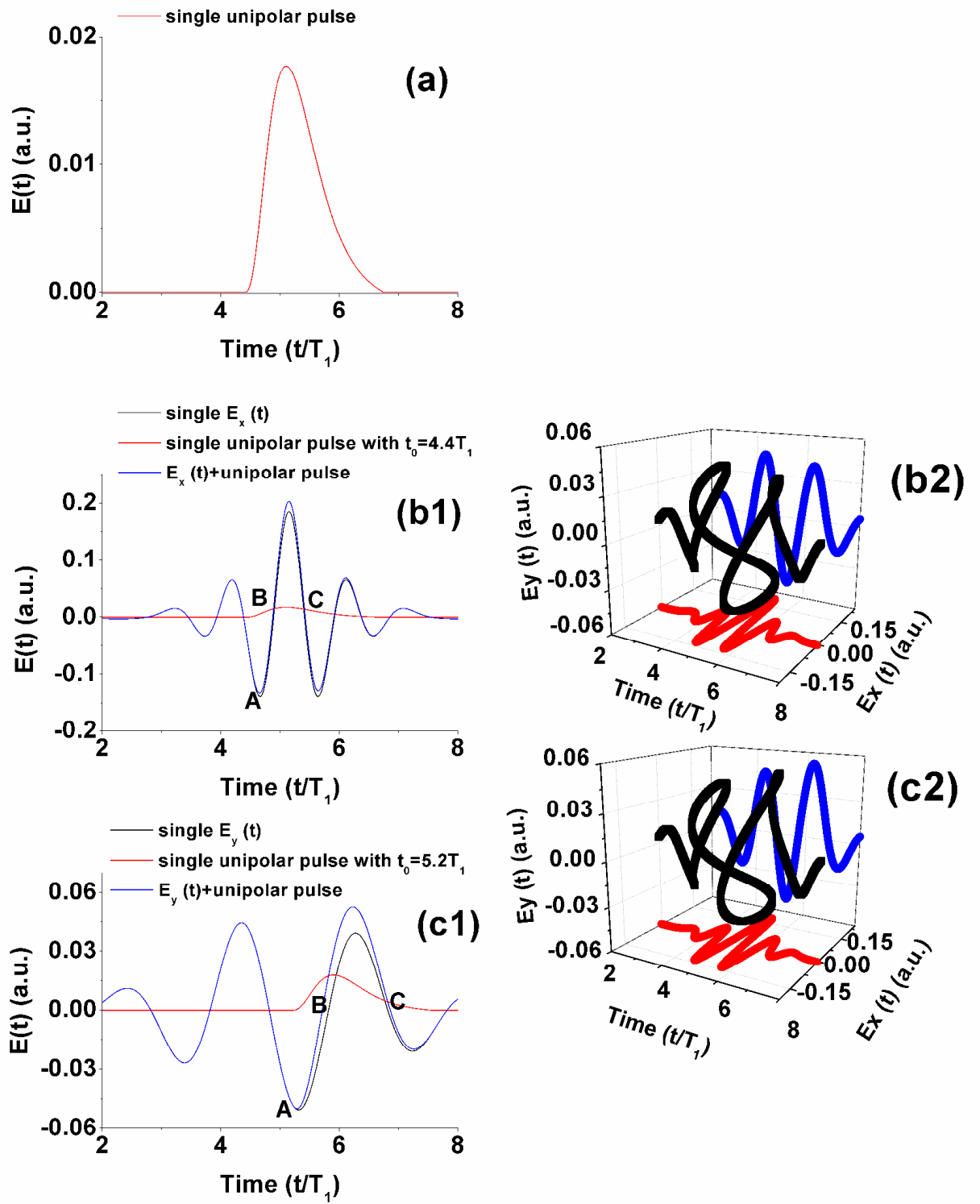

Fig. 4 


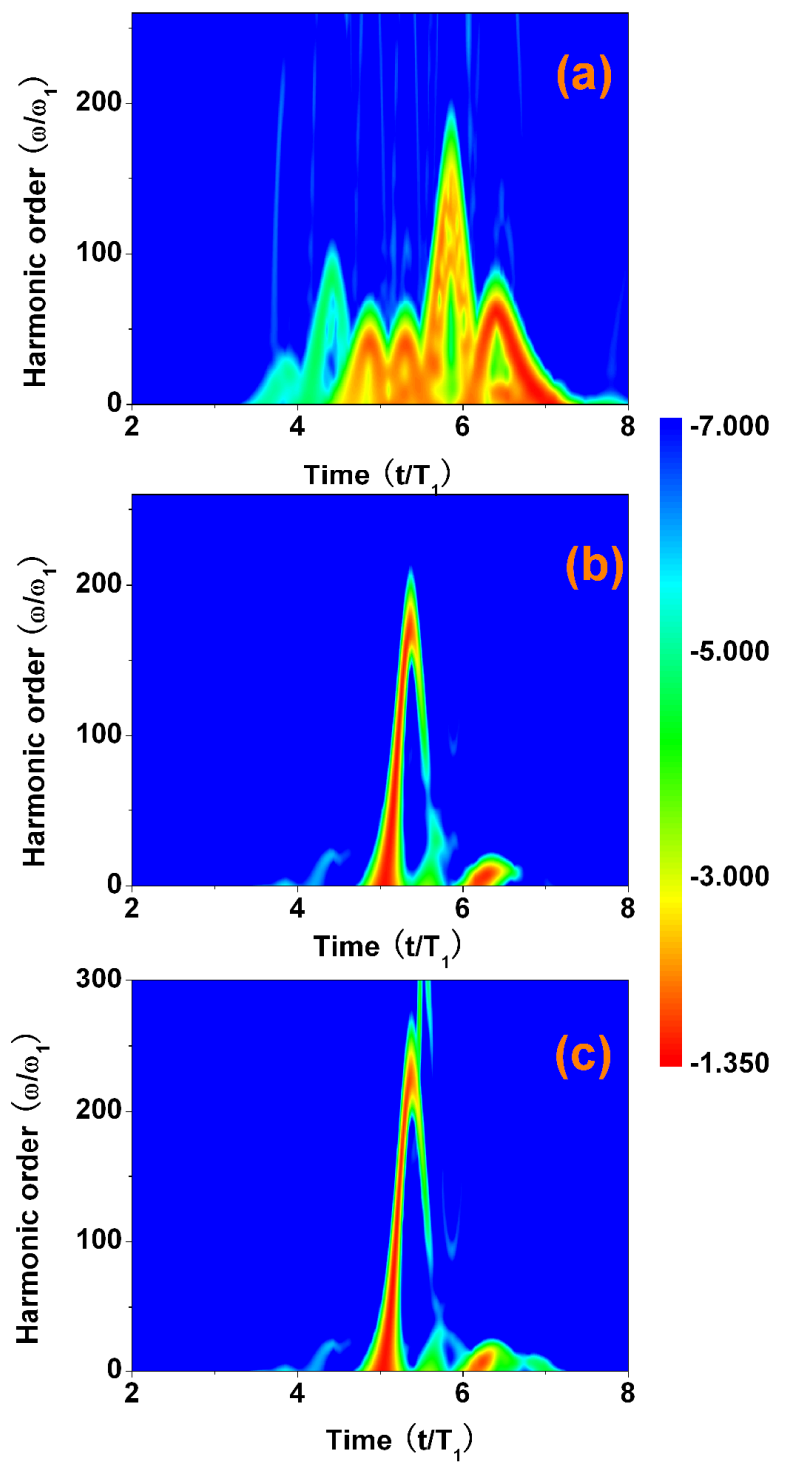

Fig. 5 

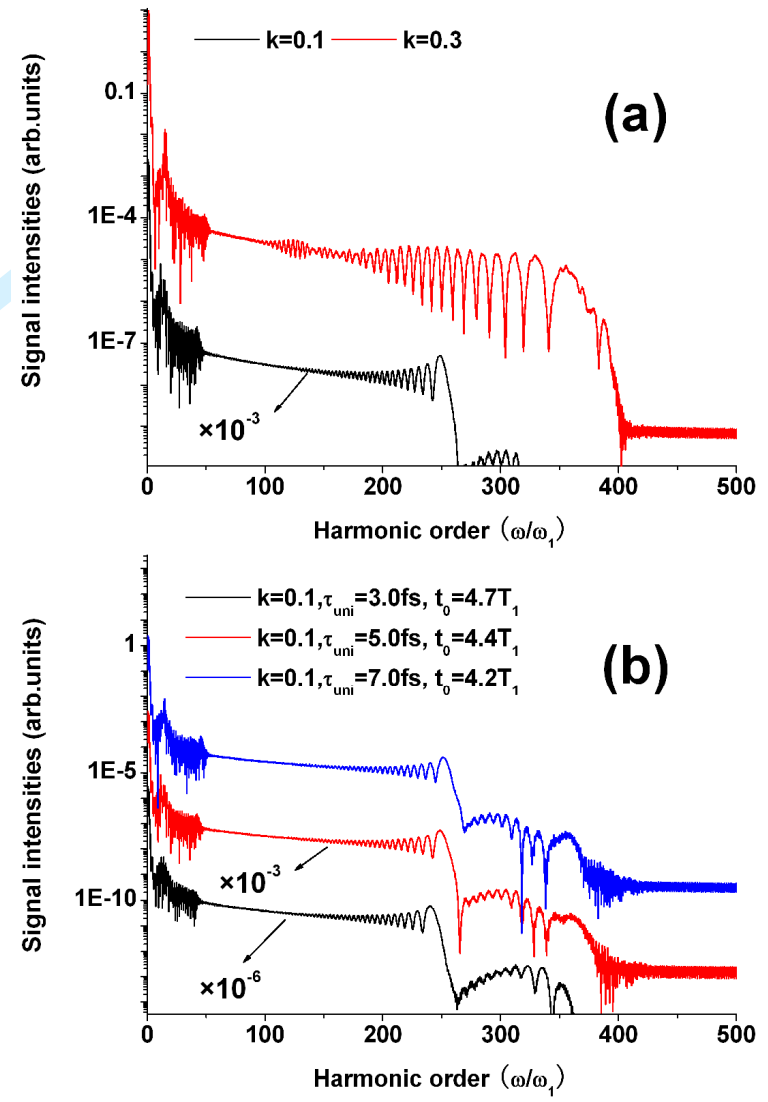

Fig. 6 


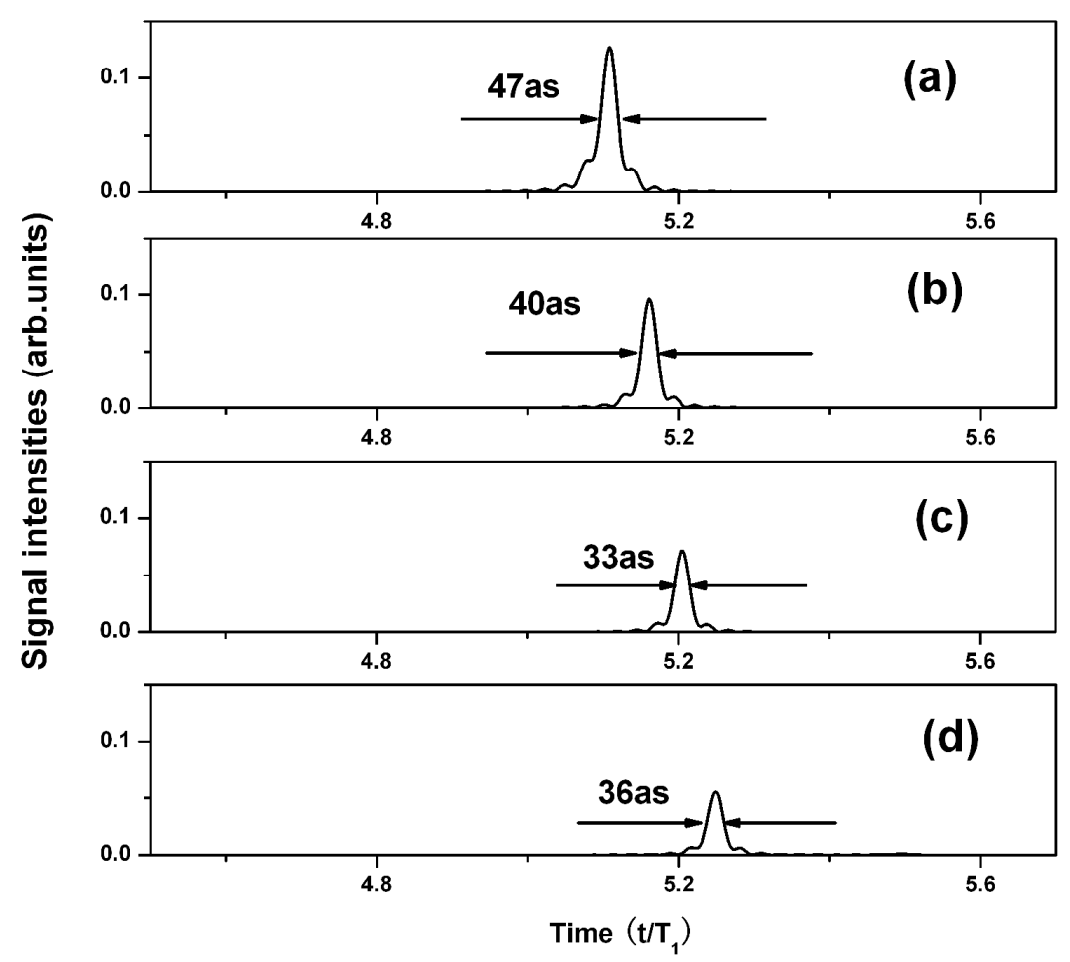

Fig. 7 